\title{
Differential immune responses of Monochamus alternatus against symbiotic and entomopathogenic fungi
}

\author{
Wei Zhang ${ }^{1,2 \dagger}$, Jie Meng ${ }^{1,2 \dagger}$, Jing Ning ${ }^{1,4 \dagger}$, Peijun Qin ${ }^{1,2}$, Jiao Zhou ${ }^{1}$, Zhen Zou ${ }^{1,2}$, Yanhong Wang ${ }^{1}$, \\ Hong Jiang ${ }^{1}$, Faheem Ahmad ${ }^{1,3}$, Lilin Zhao $^{1^{*}} \&$ Jianghua Sun $^{1,2^{*}}$ \\ ${ }^{1}$ State Key Laboratory of Integrated Management of Pest Insects and Rodents, Institute of Zoology, Chinese Academy of Sciences, Beijing \\ 100101, China; \\ ${ }^{2}$ University of Chinese Academy of Sciences, Beijing 100049, China; \\ ${ }^{3}$ Department of Biosciences, COMSATS Institute of Information Technology, Islamabad 45550, Pakistan; \\ ${ }^{4}$ College of Life Science, Hebei University, Baoding 071002, China
}

Received April 10,2017; accepted May 28,2017; published online July 28, 2017

\begin{abstract}
Monochamus alternatus, the main vector beetles of invasive pinewood nematode, has established a symbiotic relationship with a native ectotrophic fungal symbiont, Sporothrix sp. 1, in China. The immune response of M. alternatus to $S$. sp. 1 in the coexistence of beetles and fungi is, however, unknown. Here, we report that immune responses of $M$. alternatus pupae to infection caused by ectotrophic symbiotic fungus $S$. sp. 1 and entomopathogenic fungus Beauveria bassiana differ significantly. The $S$. sp. 1 did not kill the beetles while $B$. bassiana killed all upon injection. The transcriptome results showed that the numbers of differentially expressed genes in $M$. alternatus infected with $S$. sp. 1 were 2-fold less than those infected with B. bassiana at 48 hours post infection. It was noticed that Toll and IMD pathways played a leading role in the beetle's immune system when infected by symbiotic fungus, but upon infection by entomopathogenic fungus, only the Toll pathway gets triggered actively. Furthermore, the beetles could tolerate the infection of symbiotic fungi by retracing their Toll and IMD pathways at $48 \mathrm{~h}$. This study provided a comprehensive sequence resource of $M$. alternatus transcriptome for further study of the immune interactions between host and associated fungi.
\end{abstract}

Monochamus alternatus, symbiotic fungus, Beauveria bassiana, RNA-seq, immune signaling pathway

Citation: Zhang, W., Meng, J., Ning, J., Qin, P., Zhou, J., Zou, Z., Wang, Y., Jiang, H., Ahmad, F., Zhao, L., and Sun, J. (2017). Differential immune responses of Monochamus alternatus against symbiotic and entomopathogenic fungi. Sci China Life Sci 60, 902-910. doi: 10.1007/s11427-017-9102-y

\section{INTRODUCTION}

Monochamus alternatus (Cerambycidae: Coleoptera) is a species of sapro-xylophagous sawyer beetles (Vicente et al., 2012) that cause significant losses to pine trees (Alves et al., 2016). Moreover, it serves as a main vector of Bur-

$\dagger$ Contributed equally to this work

*Corresponding authors (Lilin Zhao, email: zhaoll@ioz.ac.cn; Jianghua Sun, email: sunjh@ioz.ac.cn) saphelenchus xylophilus, a causal organism of pine wilt disease (PWD), for its tree-to-tree dispersal (Mamiya and Enda, 1972). Monochamus alternatus has also developed a symbiotic relationship with an ectotrophic ophiostomatoid blue stain fungus, Sporothrix sp. 1 (Zhao et al., 2013) which also gets dispersed adhering to $M$. alternatus body surfaces (Suh et al., 2013). Unlike entomopathogenic fungi, S. sp. 1 can coexist with $M$. alternatus and is even known to promote development and survival of beetles (Zhao et al., 2013). In the nature, many insects develop symbiotic relationships 
with ophiostomatoid blue stain fungi (Dori-Bachash et al., 2015; Lu et al., 2016; Repe et al., 2013; Zhao et al., 2014). As symbiotic fungi, most of them do not kill their hosts, rather are beneficial to them (Hartley and Gange, 2009) which is very different from entomopathogenic fungi. With the symbiotic interactions concerned, the molecular mechanism of insects permitting ectotrophic fungi to coexist on their body surfaces needs to be clarified.

The symbiotic relationships between insects and microorganisms include endotrophic and ectotrophic symbiosis (Hartley and Gange, 2009). Compared to endotrophic symbiosis, the ectotrophic symbiosis is more common because of a wider range of suitable external environments and unlike the endosymbiosis, the fungi does not have to deal with the host's internal immune defense. For example, the fungus-growing ants maintain an obligate mutualism with the ectotrophic fungi they grow for food and in return, the ants provide the fungus with substrate for growth, dispersion and protection (Currie et al., 2003). Similarly, the ectotrophic symbiotic fungus Leptographium procerum of bark beetles has strong biodegrading activities to lower the strength of pine resistance toward the invasive beetle-microorganisms symbiosis (Lu et al., 2016; Xu et al., 2015). In general, most of studies on ectotrophic fungi have concentrated on symbiosis, but not on the immune responses of insets to them.

For systemic defense against pathogenic microorganisms, insects are capable to evolve their immune system to enhance cellular and humoral immunity. Upon an infection, the immune system may trigger either cellular or humoral responses against the pathogens. The cellular response is mediated by hemocytes that initiate phagocytosis, encapsulation or nodulation of pathogens (Schmidt et al., 2001; Strand and Pech, 1995) while humoral response promotes antimicrobial peptides (AMPs) production, coagulation and melanization (Gillespie et al., 1997; Lowenberger, 2001; Muta and Iwanaga, 1996). The immune signaling pathways including Toll (Valanne et al., 2011), IMD (Silverman and Maniatis, 2001), JNK (Rämet et al., 2002), and JAK/STAT (Agaisse et al., 2003) are triggered by recognition of pathogen-associated molecular patterns (PAMPs) on the microbial surface (Baeg et al., 2005). The Toll and IMD pathways are the major regulators of the immune response (De Gregorio et al., 2002; Zou et al., 2007). The activation of Toll and IMD pathways result in the expression of AMPs via NF-кB-like transcription factors (Lemaitre et al., 1996). Toll pathway is induced in response to fungal and Gram-positive bacterial infection and is mediated by an extracellular serine protease (SP) cascade. Upon infection, the Toll pathway is activated in the haemolymph and leads to the binding of Spaetzle to Toll which activates dMyD88 and the kinase Pelle. It leads to degradation of Cactus and the release of NF-KB-like transcription factors Dorsal and Dif. While, the IMD pathway is induced primarily in response to Gram-negative bacteria. The ultimate target of IMD pathway is Relish, a Rel/NF-кB transactivator. It is cleaved by the caspase Dredd and IKK. TAK, a MAPKKK, functions upstream of the IKK and downstream of IMD, a receptor interacting protein (De Gregorio et al., 2002; Lemaitre, 2004). In general, JNK and JAK/STAT pathway also could regulate AMPs (Garver et al., 2013; Park et al., 2004).

Beauveria bassiana is one of the most ubiquitous and extensively studied entomopathogenic species (Ferron, 1978). As one of the biological control agents (BCA), $B$. bassiana has high activity against a variety of agricultural pests, and essentially no toxicity or infectivity toward vertebrates (Wraight et al., 2000). When it infects the insects, $B$. bassiana elicits the cellular and humoral immune reaction of the host and activation of Toll pathway (Gottar et al., 2006; Yassine et al., 2012). Finally, this fungus overcomes the immune responses of host and kills it. Hence, in an attempt to understand the underlying mechanism of immune tolerance of host to ectotrophic symbiotic fungus, we have compared the immune responses of $M$. alternatus pupae to $S$. sp. 1 and B. bassiana. We also adopted a high-throughput RNA-seq technology without reference genome of M. alternatus to find out the nature of the immune responses of the beetles infected by different fungi. Our aim is to reveal the different immune responses of $M$. alternatus pupae to infection caused by ectotrophic symbiotic and entomopathogenic fungi and provided valuable reference information for further study of the immune interactions between the host and symbiotic fungi.

\section{RESULTS}

\section{The death rates of $M$. alternatus infected by different fungi}

The pupae of $M$. alternates displayed different responses upon infection by $S$. sp. 1 and B. bassiana in injection and contact experiments. The results demonstrated no significant differences in development and responses of the pupae infected with $S$. sp. 1 and the control. Both type of pupae continued their normal development and became adults. However, pupae that were injected with $B$. bassiana turned black in color around the wound within $24 \mathrm{~h}$, and after $48 \mathrm{~h}$ of infection only $23 \%$ of pupae could survive. At $72 \mathrm{~h}$ after infection, these pupae were completely melanized and died. A month later, these carcass were completely wrapped in fungal mycelium and conidial masses (Figure 1A and B).

Similar results were observed in contact experiment (Figure 1C). In 5 days, the mycelia hyphae of both $S$. sp. 1 and $B$. bassiana started invading the bodies of pupae. However, at the 10 days, the pupae in $B$. bassiana cultures died and were completely wrapped in mycelia mass. Whereas in contrast to that, the pupae kept in $S$. sp. 1 cultures were alive and continued their normal development until adult eclosion. 

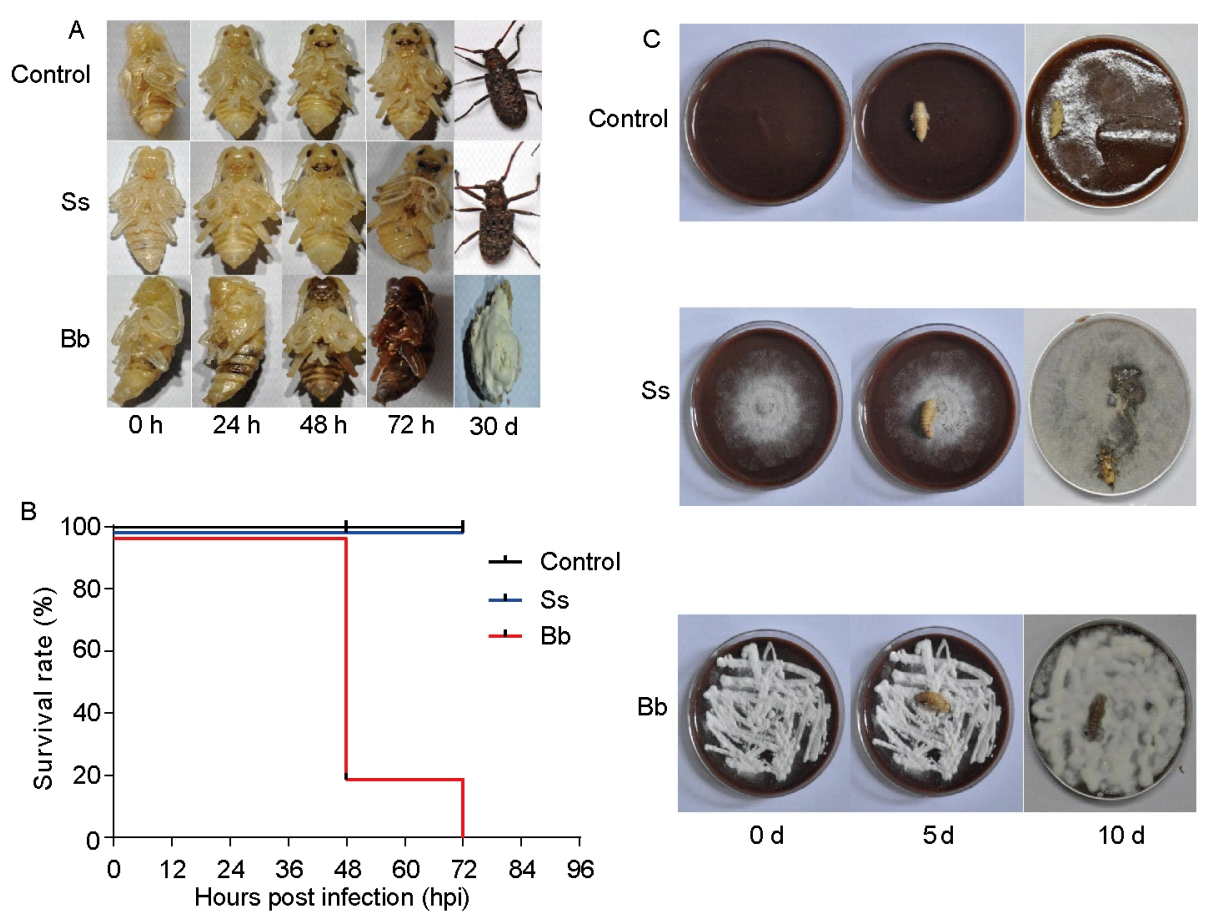

Figure 1 Differential survival response of M. alternatus infected by B. bassiana and $S$. sp. 1. A, Different phenotypes of the M. alternatus pupae infected with Sporothrix sp. 1 and B. bassiana. The infection of $S$. sp. 1 would not cause death to M. alternatus and beetles could develop into adult beetles, while the infection of B. bassiana would lead to the death of beetles after 24 hours post injection. B, Survival curves of M. alternatus pupae infected by $S$. sp. 1 (blue line) and B. bassiana (red line) compared with the control (black line). Each experiment was repeated three times. C, Different phenotypes of the M. alternatus pupae in contact with $S$. sp. 1 and B. bassiana.

\section{Identification and functional classification of differential expressed transcripts of M. alternatus injected by differ- ent fungi}

To find out the different death rates of $M$. alternatus infected by different fungi, the cDNA libraries of infected $M$. alternatus were constructed and the differentially expressed genes were analyzed. According to BLASTX searches against the non-redundant (NR) sequence database, 55,059 unigenes were annotated. The profiles of 48 h post-infection by $S$. sp. 1 and no-infection (control) clustered together with similar expression patterns, indicating that pupae adapted to the symbiotic fungus $S$. sp. 1 and maintained a normal body state. In addition, five major gene clusters were identified that exhibited a distinct expression pattern over time (Figure 2A). The up-regulations of 19,151 unigenes were observed in cluster 1 , showing a severe response of $M$. alternatus to B. bassiana infection after $24 \mathrm{~h}$. However, after $48 \mathrm{~h}$ of $B$. bassiana infection, other cluster genes (cluster 3, 11,969 unigenes) were up-regulated. A large number of genes $(9,576)$ in cluster 2 were expressed mostly after $24 \mathrm{~h}$ infection by $S$. sp. 1. However, most genes showed normal expression after $48 \mathrm{~h}$ infection except cluster 4 genes $(6,702)$. These results suggested that the gene regulations were different between the fungi infections and the pupae after $48 \mathrm{~h}$ infection by $S$. sp. 1 tended to be normal.

In comparison to control group, the results show that 10,122 transcripts were significantly regulated (5,453 up- and
4,669 down-regulated) $24 \mathrm{~h}$ after infection by $B$. bassiana, while more transcripts (8,406 up- and 5,309 down-regulated transcripts) were changed at $48 \mathrm{~h}$. With the increase of the infection time, the gene expression of beetles became active. There were 5,967 commonly regulated transcripts $(2,605+239+298+2,825)$ at both time points injected by $B$. bassiana (Figure 2B). For $S$. sp. 1 infected pupae, a total of 5,111 transcripts were up-regulated and 4,179 were down-regulated at 24 hours post injection, while fewer transcripts (2,493 up- and 4,267 down-regulated) were significantly changed at $48 \mathrm{~h}$ after infection (Figure $2 \mathrm{C}$ ). These results indicate that the gene regulation of beetles was active at $24 \mathrm{~h}$ after infection by both $S$. sp. 1 and B. bassiana, however, the expression varied significantly at $48 \mathrm{~h}$ after infection between pupae infected by different fungi. The gene regulation of $M$. alternatus infected by $B$. bassiana was more active at $48 \mathrm{~h}$ (up 5,503+2,605+298, down 2,245+239+2,825), while that infected by $S$. sp. 1 was less active at $48 \mathrm{~h}$ (up $1,045+1,309+139$, down $1,852+281+2,134)$. These results corresponded to the heat map cluster of the transcriptional FPKM values. The data of $\mathrm{Bb}$ and Ss groups at $24 \mathrm{~h}$ were clustered together, and then clustered with the most active $\mathrm{Bb}$ group at $48 \mathrm{~h}$, meanwhile the data of Ss group at $48 \mathrm{~h}$ and control were clustered (Figure 2A).

To further explore the potential functions of identified DETs and the pathways involved, we performed the Gene Ontology (GO) enrichment and KEGG analysis for DETs. 


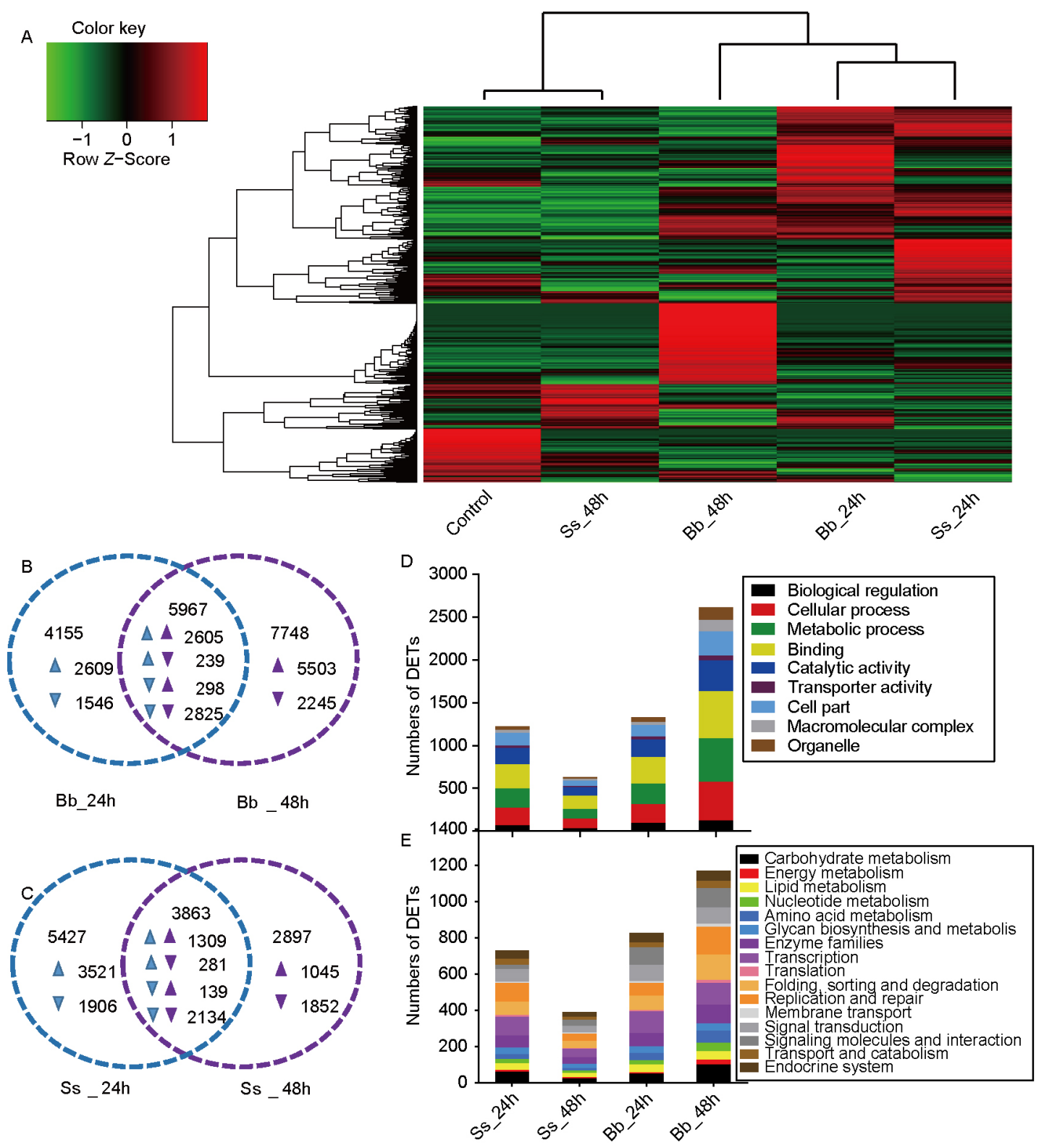

Figure 2 The transcriptome differences of $M$. alternatus infected by $S$. sp. 1 and B. bassiana. A, Hierarchical cluster analysis of differentially expressed transcripts (DETs) obtained from transcriptomes of $S$. sp. 1 and B. bassiana infected M. alternatus, 24 and 48 hours post infection. B, A Venn diagram of Statistics of DETs of B. bassiana injected after 24 and 48 h. C, Statistics of DETs of $S$. sp. 1 injected after 24 and 48 h. D, Distribution of GO function groups within DEGs in $S$. sp. 1 and B. bassiana-infected pupae. E, Distribution of KEGG function groups within up-regulated genes in $S$. sp. 1 and B. bassiana infected pupae.

There were three parts of the GO function annotations, including cellular component, molecular function and biological process (Ashburner et al., 2000). Results showed that most of DETs were enriched in molecular function (catalytic activity, binding) and biological process (metabolic process, cellular process) (Figure 2D). KEGG pathway analysis of up-regulated DETs in $\mathrm{Ss}$ and $\mathrm{Bb}$ groups revealed that more DETs were involved in transcription, folding, sorting and degradation, replication and repair, carbohydrate metabolism, enzyme families, signal transduction, signaling molecules and interaction (Figure 2E). The results of GO and KEGG analysis indicated that fungal infections affected the cellular response and the overall metabolic activities of the insects. The gene regulation of beetles infected by $B$. bassiana at $48 \mathrm{~h}$ was most active, while that by $S$. sp. 1 at $48 \mathrm{~h}$ was least active which tended to be non-infection conditions for beetles.

\section{The classification and expression profile of immunity-re- lated genes of $M$. alternatus}

BLASTX searches annotated 197 immunity-related genes of $M$. alternatus from 55,059 unigenes. These genes included pathogen recognition molecules, signal modulation molecules, intracellular signal transduction molecules, im- 


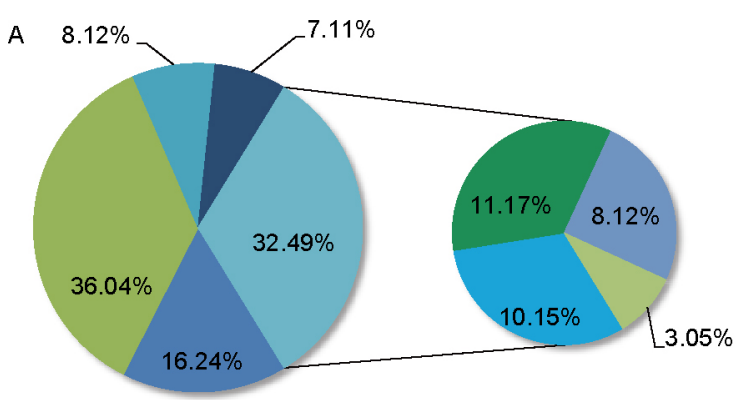

\begin{tabular}{|l|}
\hline Pathogen recognition molecules \\
Signal modulation molecules \\
Immune response effectors \\
Cellular immunity genes \\
Toll pathway \\
IMD pathway \\
JNK pathway \\
JAK/STAT pathway
\end{tabular}

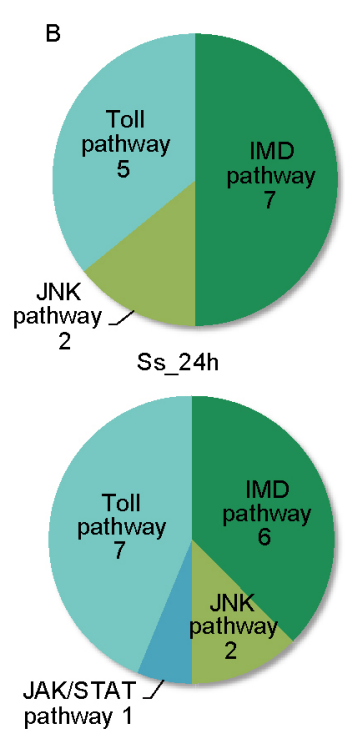

Bb_24h

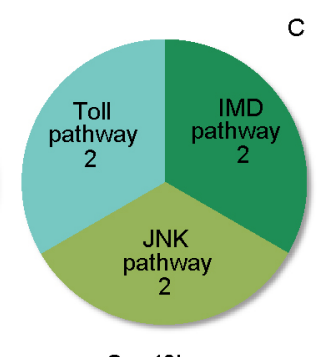

Ss_48h

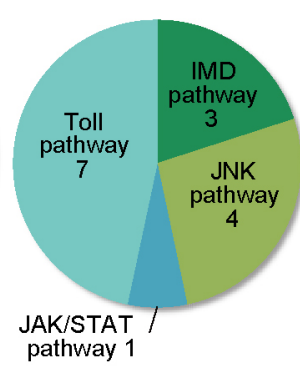

Bb_48h

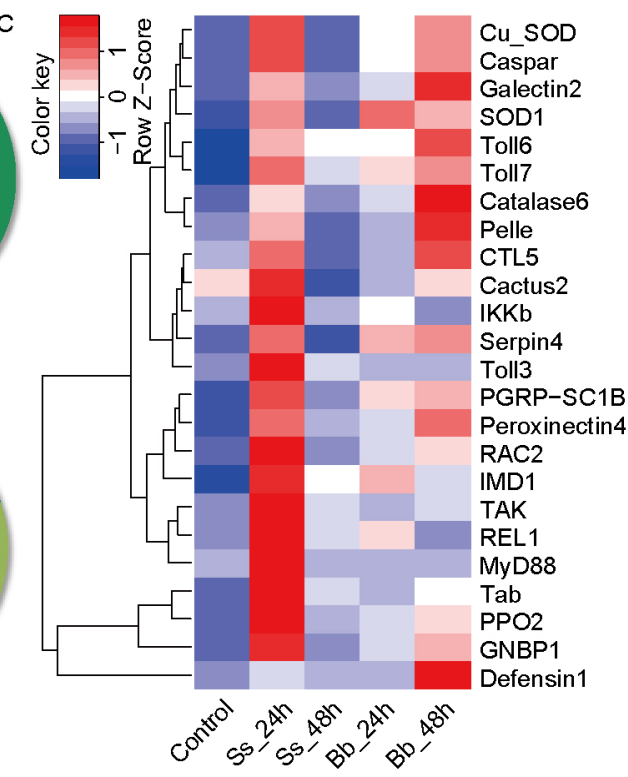

Figure 3 Characterization of immunity-related genes of $M$. alternatus. A, Distribution of M. alternatus immunity-related transcripts in categories of pathogen recognition, signal modulation, intracellular signal transduction, immune response effector and cellular immunity. B, Distribution of DETs in intracellular signaling pathways of $S$. sp. 1 and B. bassiana infected M. alternatus, 24 and 48 hours post infection. C, Hierarchical cluster analysis of $M$. alternatus main differential expression genes. Fat body samples were divided into three groups: $S$. sp. 1 group, B. bassiana group and the control. Blue, white and red colors represent low, intermediate and high relative mRNA expression level.

mune response effectors and cellular immune genes. The group of intracellular signal transduction molecules about $32.49 \%$ was related to Toll, IMD, JNK and JAK/STAT pathways. Among this group we identified 20 immunity-related genes in Toll pathway and 22 immunity-related genes in IMD pathway that were the main signal pathways in insect immunity (Figure 3A).

Hence the intracellular signal transduction molecules, including genes of Toll and IMD pathway, were filtered out from differentially expressed genes of the transcriptomes compared to control group. After 24 hours of infection, 14 genes were detected from the DETs of M. alternatus infected by $S$. sp. 1 which mainly included the genes for Toll and IMD pathways. After $48 \mathrm{~h}$ of infection by $S$. sp. 1, the number of DETs decreased quickly, especially the number of genes for Toll and IMD pathway (Table S1 in Supporting Information). In contrast, when the pupae were infected by B. bassiana, the number of DETs was higher after $24 \mathrm{~h}$ which was 16 . But after $48 \mathrm{~h}$, the number of DETs in intracellular signal transduction of Ss group (6) was not like Bb group (15). The number of DETs in intracellular signal transduction of beetles infected by $B$. bassiana after $24 \mathrm{~h}$ was similar to that after $48 \mathrm{~h}$ and the Toll pathway occupied a dominant position (Figure 3B).

\section{Quantitative real-time PCR analysis of selected immune- related genes}

In addition, we performed quantitative real-time PCR analysis of selected immunity-related genes to verify the immune response changes between control and treatment groups. The results revealed that key genes of $S$. sp. 1 infected pupae in Recognition, Toll (Toll, MyD88, Pelle, Cactus) and IMD (IMD, Tab, Tak, Ikkb, Rel) pathways maintained a high level of expression at 24 hours post infection. But the expression of these immunity-related genes returned to normal levels at $48 \mathrm{~h}$ after infection resulting to immune tolerance of $M$. alternatus to $S$. sp. 1. However, in the pupae infected by $B$. bassiana, the expression levels of immunity-related genes 
changed unconspicuously at $24 \mathrm{~h}$. Noticeably, the genes in recognition and Toll pathway were active very significantly, whereas the genes in IMD pathway were almost not active at $48 \mathrm{~h}$ (Figure 3C).

\section{DISCUSSION}

The interaction of symbiotic microorganism and their hosts is beneficial to maintain physiology, metabolism and immune homeostasis of hosts (Bäckhed et al., 2005). Also the innate immunity of hosts can maintain the delicate balance of immune tolerance in the symbiotic system. Although the symbiotic relationships exist in most of the metazoan, immune mechanism of ectotrophic symbiosis is still not very clear. Here we found the immune tolerance of the beetle $M$. alternatus to the infection caused by ectotrophic symbiont $S$. sp. 1. According to the immunity-related genes expression profile of $S$. sp. 1 infected pupae at different time points, blue stain fungus triggered an acute immune response in the early stage of infection, whereas M. alternatus tolerated the infection of $S$. sp. 1 and maintained a normal body state at later stage of infection, the heat map of expressions of immunity-related genes was clustered similarly with control groups. Coincidentally, this is obvious in the intestinal environment of organisms where the associated microorganisms forms a set of sophisticated immune mechanism to tolerate the microorganisms in symbiosis (Müller et al., 2005; Macdonald and Monteleone, 2005). An immune regulator of Drosophila, PGRP-LC-interacting inhibitor of IMD signaling (PIMS), decreases the release of peptidoglycan recognition protein (PGRP-LC) from the plasma membrane hence block the IMD pathway of Drosophila to tolerate the symbiosis of bacteria (Lhocine et al., 2008). In our research, a similar immune tolerance mechanism has been observed when insects maintain symbiosis with endotrophic fungi. The larva of ghost moth, Hepialus xiaojinensis, developed tolerance to the endotrophic fungus, Ophiocordyceps sinensis. The heatmap of $H$. xiaojinensis infected by $O$. sinensis showed normal expression levels after prolonged infection (Meng et al., 2015). However, most of researches focus on the endotrophic system, while our studies showed the immune responses of host infected by an ectotrophic symbiont.

As symbiotic fungi, most of them do not kill their host at the early stage, even are beneficial to their hosts (Hartley and Gange, 2009). This is very different from entomopathogenic fungi, such as B. bassiana. Our research show that at $24 \mathrm{~h}$, the immune responses of $M$. alternatus infected by $B$. bassiana were inactive. However, the genes of Toll pathway were activated vigorously at $48 \mathrm{~h}$, and the beetles were dead within a few days. Similarly, previous research suggested that natural infection of Drosophila by B. bassiana leads to the expression, via the selective activation of genes in Toll pathway encoding antifungal activity (Lemaitre et al., 1997). While the host showed different immune responses to symbiotic fungi. The immune responses of $M$. alternatus infected by symbiont $S$. sp. 1 were activated in both IMD and Toll pathways. However, these immunity-related genes were inactive at $48 \mathrm{~h}$, which could maintain the symbiosis between host and symbiotic microorganism.

Many studies have reported that the presence of ophiostomatoid blue stain fungi, Ophiostoma minus and Trichoderma sp., increased the number of PWNs carried by M. alternatus (Maehara and Futai, 2000) and S. sp. 1 also had a positive influence on the growth and survival of beetles (Zhao et al., 2013). At the transcriptome level, we noticed activation of 9,290 transcripts of beetles infected by $S$. sp. 1 after 24 h. Besides, most of DETs belonged to molecular function and biological process, a majority of up-regulated genes were mainly involved in transcription, folding, sorting and degradation, replication and repair, carbohydrate metabolism, signal transduction, signaling molecules and interaction, etc. Briefly, we could speculate that $S$. sp. 1 might accelerate the metabolism of $M$. alternatus and the injection of $S$. sp. 1 could cause immune responses and conspicious changes on physiological metabolism in beetles. The immune tolerance of M. alternatus might be related to the positive influence of $S$. sp. 1 to the development of $M$. alternatus, which should be clarified in the future.

The research of the interaction between $M$. alternatus and the native fungal symbiont, $S$. sp. 1 is an important part of the symbiotic system that contains $B$. xylophilus, $M$. alternatus and native ophiostomatoid blue stain fungi. However, $M$. alternatus genome is still unavailable, and hence is a huge obstacle to understanding the molecular biology of beetles in relation to B. xylophilus and blue stain fungi. In this research, 55,059 unigenes and 197 immunity-related genes, including many key genes in Toll and IMD pathways were annotated from RNA-seq. The genes identified and annotated in this study will contribute to a better knowledge of molecular basis of immunity of beetles against its symbiotic fungi, and ultimately provides a new direction to study pinewood disease system for better understanding of the interaction of forest pests and fungi.

\section{MATERIALS AND METHODS}

\section{Beetle rearing and fungi culture}

Monochamus alternatus beetles were collected from Zhejiang Province, China and their cultures were maintained in the laboratory for several generations before experiments. The larvae were reared on an artificial diet at $25^{\circ} \mathrm{C}$ in the dark and used in bioassays at the early pupal stage.

The fungal cultures were obtained on potato dextrose agar (PDA) plates at $25^{\circ} \mathrm{C}$ and $80 \%$ humidity for one week. Sporothrix sp. 1 (CMW29982) is a local Chinese species of ophiostomatoid blue stain fungi and the specimen is 
deposited in the culture collection (CMW) of the Forestry and Agricultural Biotechnology Institute (FABI), University of Pretoria, Pretoria, South Africa. The strain number of $B$. bassiana is ARSEF2860.

\section{Survival analysis of co-culture of beetles and fungi}

In order to be closer to the actual condition in the field for the co-culture tests, phloem media containing mixture of bast powder and MEA was used to culture $S$. sp. 1 or B. bassiana in this experiment. At the first day of pupation, M. alternatus pupae (10 in each group) were cultured on the plates of $S$. sp. 1 or B. bassiana. Next, the plates were incubated at $25^{\circ} \mathrm{C}$ in the dark and checked daily for the growth of beetles. Pupal survival was counted daily after infection until they died to calculate mortality rate. The experiment was repeated three times. GraphPad software was used in all statistical analysis.

\section{Survival analysis of beetles injected by fungi and RNA sample preparation}

At the first day of pupation, beetles from the same batch were divided into three groups (10 beetles in each group). Each pupa was infected with $S$. sp. 1, B. bassiana or sterile PBST (as control) by injecting $2 \mu \mathrm{L}$ diluted fungal suspension $\left(3 \times 10^{6}\right.$ conidia $\left.\mu \mathrm{L}^{-1}\right)$ at second abdominal segment using a glass capillary injector. The infected pupae were incubated at $25^{\circ} \mathrm{C}$. Pupal survival was observed at 24,48 and $72 \mathrm{~h}$ and mortality rates were calculated.

For RNA sampling, fat body tissues were collected under a dissection microscope from pupae 24 and $48 \mathrm{~h}$ after they were infected with $S$. sp. 1 and B. bassiana. Control samples from PBST-injected pupae were prepared at $48 \mathrm{~h}$ after infection in the same way. Samples were lysed in $1 \mathrm{~mL}$ of TRizol Reagent (Invitrogen, USA) to extract total RNA according to the manufacturer's instructions. RNA concentrations were detected on a ND-1000 Spectrophotometer (NanoDrop Technologies, Inc., USA). Each sample was prepared by combining three individual beetles.

\section{Library construction and Illumina sequencing}

From each treatment or control group, total RNA of $5 \mu \mathrm{g}$ was used to enrich poly (A) mRNA using oligo (dT) magnetic beads Dynabeads ${ }^{\circledR}$. RNA-seq libraries were prepared by following the RNA-Seq Library Preparation Kit for Whole Transcriptome Discovery (Wang et al., 2015). The treatment and control libraries were sequenced on Illumina HiSeq 2000 platform in the Beijing Institute of Genomics (Chinese Academy of Sciences).

\section{Assembly of transcriptomes and functional classification}

The original sequences from each cDNA library were processed to remove low quality sequences, adaptors and readings contaminated with microbes. The clean sequences were, then, de novo assembled to produce contigs using the short reads assembling program, Trinity (version 2013), in accordance with default parameters. After assembly and removal of redundant sequences, the clean reads were clustered using the Cd-hit clustering software. For functional annotations, we searched all unigenes against the NCBI non-redundant (NR) sequence database (http://www.ncbi.nlm.nih.gov/) and Swiss-prot database using BLASTX $\left(E\right.$-value $\left.<10^{-5}\right)$. Moreover, in order to confirm enriched $\mathrm{GO}$ terms, all unigenes were subjected to gene ontology (GO) enrichment analysis through BLAST2GO against GO database. Finally, based on the KEGG (Kyoto Encyclopedia of Genes and Genomes database) analysis, we detected the enriched pathway and metabolic networks at the KEGG website (Hou et al., 2015). The R package RSEM was used to calculate the fragments per kilobase of exon per million fragments mapped (FPKM) value (Tu et al., 2012). The differentially expressed genes (DEGs) were filtered out by R package DEGseq with a cut-off $P$-value $<0.001$ (Wang et al., 2010). The genes were considered differentially or significantly expressed if they had a $P$ value $<0.05$ and a fold change of either $>2$ or $<0.5$ (Xiong et al., 2015).

\section{Quantitative real-time PCR analysis and hierarchical clustering of main differentially expressed transcripts (DETs)}

To confirm the expression profile from RNA-seq results, we selected some of the major immunity-related genes and designed specific primers (Table S2 in Supporting Information) to perform a quantitative real-time PCR analysis. Total RNA of $1 \mu \mathrm{g}$ was used for cDNA synthesis with FastQuant RT Kit (Tiangen, Beijing). The quantitative real-time PCR was performed on a MX3000P system using SYBR green PCR Master Mix. The thermal cycling conditions were: $95^{\circ} \mathrm{C}$, $5 \mathrm{~s} ; 55^{\circ} \mathrm{C}, 30 \mathrm{~s} ; 72^{\circ} \mathrm{C}, 30 \mathrm{~s}$ ( 40 cycles). The qPCR data were collected and analyzed by Hierarchical cluster analysis performed using heatmap.2 function within gplots package in $\mathrm{R}$ environment.

\section{Statistical analysis}

In all experiments, the data between the two groups were evaluated by the unpaired two-tailed Student's $t$ test or MannWhitney $U$ test depending on the results of the tests of normality and homogeneity. The data obtained were analyzed using SPSS 18.0 software (SPSS, Inc., USA) (Vandepitte et al., 2014).

Compliance and ethics The author(s) declare that they have no conflict of interest.

Acknowledgements This work was supported by the Forestry Industry Research Special Funds for Public Welfare Project (201204501), National Key 
Plan for Scientific Research and Development of China (2016YFC1200604, 2016YFD0500300), the High Technology Research and Development Program (HTRDP) of China (2014AA020529), National Natural Science Foundation of China (31572272, 31370650, 31402013, 31221091, 31672291, L1524009), the CAS Key Research Projects of the Frontier Science (QYZDBSSW-SMC014), and CAS (2015-SM-C-02).

Agaisse, H., Petersen, U.M., Boutros, M., Mathey-Prevot, B., and Perrimon, N. (2003). Signaling role of hemocytes in Drosophila JAK/STAT-dependent response to septic injury. Dev Cell 5, 441-450.

Alves, M., Pereira, A., Matos, P., Henriques, J., Vicente, C., Aikawa, T., Hasegawa, K., Nascimento, F., Mota, M., Correia, A., and Henriques, I. (2016). Bacterial community associated to the pine wilt disease insect vectors Monochamus galloprovincialis and Monochamus alternatus. Sci Rep 6, 23908.

Ashburner, M., Ball, C.A., Blake, J.A., Botstein, D., Butler, H., Cherry, J.M., Davis, A.P., Dolinski, K., Dwight, S.S., Eppig, J.T., Harris, M.A., Hill, D.P., Issel-Tarver, L., Kasarskis, A., Lewis, S., Matese, J.C., Richardson, J.E., Ringwald, M., Rubin, G.M., and Sherlock, G. (2000). Gene Ontology: tool for the unification of biology. Nat Genet 25, 25-29.

Bäckhed, F., Ley, R.E., Sonnenburg, J.L., Peterson, D.A., and Gordon, J.I. (2005). Host-bacterial mutualism in the human intestine. Science 307 , 1915-1920.

Baeg, G.H., Zhou, R., and Perrimon, N. (2005). Genome-wide RNAi analysis of JAK/STAT signaling components in Drosophila. Genes Dev 19, 1861-1870.

Currie, C.R., Wong, B., Stuart, A.E., Schultz, T.R., Rehner, S.A., Mueller, U.G., Sung, G.H., Spatafora, J.W., and Straus, N.A. (2003). Ancient tripartite coevolution in the attine ant-microbe symbiosis. Science 299, 386-388.

De Gregorio, E., Spellman, P.T., Tzou, P., Rubin, G.M., and Lemaitre, B. (2002). The Toll and Imd pathways are the major regulators of the immune response in Drosophila. EMBO J 21, 2568-2579.

Dori-Bachash, M., Avrahami-Moyal, L., Protasov, A., Mendel, Z., and Freeman, S. (2015). The occurrence and pathogenicity of Geosmithia spp. and common blue-stain fungi associated with pine bark beetles in planted forests in Israel. Eur J Plant Pathol 143, 627-639.

Ferron, P. (1978). Biological control of insect pests by entomogenous fungi. Annu Rev Entomol 23, 409-442.

Garver, L.S., de Almeida Oliveira, G., and Barillas-Mury, C. (2013). The JNK pathway is a key mediator of Anopheles gambiae antiplasmodial immunity. PLoS Pathog 9, e1003622.

Gillespie, J.P., Kanost, M.R., and Trenczek, T. (1997). Biological mediators of insect immunity. Annu Rev Entomol 42, 611-643.

Gottar, M., Gobert, V., Matskevich, A.A., Reichhart, J.M., Wang, C., Butt, T.M., Belvin, M., Hoffmann, J.A., and Ferrandon, D. (2006). Dual detection of fungal infections in Drosophila via recognition of glucans and sensing of virulence factors. Cell 127, 1425-1437.

Hartley, S.E., and Gange, A.C. (2009). Impacts of plant symbiotic fungi on insect herbivores: mutualism in a multitrophic context. Annu Rev Entomol 54, 323-342.

Hou, Y., Wang, X.L., Saha, T.T., Roy, S., Zhao, B., Raikhel, A.S., and Zou, Z. (2015). Temporal coordination of carbohydrate metabolism during mosquito reproduction. PLoS Genet 11, e1005309.

Lemaitre, B. (2004). The road to Toll. In: Nature Reviews Immunology. (New York: Nature Publishing), pp. 521-527.

Lemaitre, B., Nicolas, E., Michaut, L., Reichhart, J.M., and Hoffmann, J.A. (1996). The dorsoventral regulatory gene cassette spätzle/Toll/cactus controls the potent antifungal response in Drosophila adults. Cell 86, 973-983.

Lemaitre, B., Reichhart, J.M., and Hoffmann, J.A. (1997). Drosophila host defense: differential induction of antimicrobial peptide genes after infection by various classes of microorganisms. Proc Natl Acad Sci USA 94, 14614-14619.
Lhocine, N., Ribeiro, P.S., Buchon, N., Wepf, A., Wilson, R., Tenev, T., Lemaitre, B., Gstaiger, M., Meier, P., and Leulier, F. (2008). PIMS modulates immune tolerance by negatively regulating Drosophila innate immune signaling. Cell Host Microbe 4, 147-158.

Lowenberger, C. (2001). Innate immune response of Aedes aegypti. Insect Biochem Mol Biol 31, 219-229.

Lu, M., Hulcr, J., and Sun, J. (2016). The role of symbiotic microbes in insect invasions. Annu Rev Ecol Evol Syst 47, 487-505.

Müller, C.A., Autenrieth, I.B., and Peschel, A. (2005). Intestinal epithelial barrier and mucosal immunity. CMLS Cell Mol Life Sci 62, 1297-1307.

Macdonald, T.T., and Monteleone, G. (2005). Immunity, inflammation, and allergy in the gut. Science 307, 1920-1925.

Maehara, N., and Futai, K. (2000). Population changes of the pinewood nematode, Bursaphelenchus xylophilus (Nematoda: Aphelenchoididae), on fungi growing in pine-branch segments. Appl Entomol Zool 35, 413-417.

Mamiya, Y., and Enda, N. (1972). Transmission of Bursaphelenchus Lignicolus (Nematoda: Aphelenchoididae) by Monochamus Alternatus (Coleoptera: Cerambycidae). Nematologica 18, 159-162.

Meng, Q., Yu, H.Y., Zhang, H., Zhu, W., Wang, M.L., Zhang, J.H., Zhou, G.L., Li, X., Qin, Q.L., Hu, S.N., and Zou, Z. (2015). Transcriptomic insight into the immune defenses in the ghost moth, Hepialus xiaojinensis, during an Ophiocordyceps sinensis fungal infection. Insect Biochem Mol Biol 64, 1-15.

Muta, T., and Iwanaga, S. (1996). The role of hemolymph coagulation in innate immunity. Curr Opin Immunol 8, 41-47.

Park, J.M., Brady, H., Ruocco, M.G., Sun, H., Williams, D.A., Lee, S.J., Kato, T., Richards, N., Chan, K., Mercurio, F., Karin, M., and Wasserman, S.A. (2004). Targeting of TAK1 by the NF- B protein Relish regulates the JNK-mediated immune response in Drosophila. Genes Dev 18, 584-594.

Rämet, M., Lanot, R., Zachary, D., and Manfruelli, P. (2002). JNK signaling pathway is required for efficient wound healing in Drosophila. Dev Biol 241, 145-156.

Repe, A., Kirisits, T., Piškur, B., de Groot, M., Kump, B., and Jurc, M. (2013). Ophiostomatoid fungi associated with three spruce-infesting bark beetles in Slovenia. Ann For Sci 70, 717-727.

Schmidt, O., Theopold, U., and Strand, M. (2001). Innate immunity and its evasion and suppression by hymenopteran endoparasitoids. Bioessays 23, 344-351.

Silverman, N., and Maniatis, T. (2001). NF-kappaB signaling pathways in mammalian and insect innate immunity. Genes Dev 15, 2321-2342.

Strand, M.R., and Pech, L.L. (1995). Immunological basis for compatibility in parasitoid-host relationships. Annu Rev Entomol 40, 31-56.

Suh, D.Y., Hyun, M.W., Kim, J.J., Son, S.Y., and Kim, S.H. (2013). Ophiostoma ips from pinewood nematode vector, Japanese pine sawyer beetle (Monochamus alternatus), in Korea. Mycobiology 41, 59-62.

Tu, Q., Cameron, R.A., Worley, K.C., Gibbs, R.A., and Davidson, E.H. (2012). Gene structure in the sea urchin Strongylocentrotus purpuratus based on transcriptome analysis. Genome Res 22, 2079-2087.

Valanne, S., Wang, J.H., and Rämet, M. (2011). The Drosophila toll signaling pathway. J Immunol 186, 649-656.

Vandepitte, K., de Meyer, T., Helsen, K., van Acker, K., Roldán-Ruiz, I., Mergeay, J., and Honnay, O. (2014). Rapid genetic adaptation precedes the spread of an exotic plant species. Mol Ecol 23, 2157-2164.

Vicente, C., Espada, M., Vieira, P., and Mota, M. (2012). Pine Wilt Disease: a threat to European forestry. Eur J Plant Pathol in press doi: 10.1007/s10658-012-9979-3.

Wang, L., Feng, Z., Wang, X., Wang, X., and Zhang, X. (2010). DEGseq: an $R$ package for identifying differentially expressed genes from RNA-seq data. Bioinformatics 26, 136-138.

Wang, Y.H., Hu, Y., Xing, L.S., Jiang, H., Hu, S.N., Raikhel, A.S., and Zou, Z. (2015). A critical role for CLSP2 in the modulation of antifungal immune response in mosquitoes. PLoS Pathog 11, e1004931.

Wraight, S.P., Carruthers, R.I., Jaronski, S.T., Bradley, C.A., Garza, C.J., and Galaini-Wraight, S. (2000). Evaluation of the entomopathogenic 
fungi Beauveria bassiana and Paecilomyces fumosoroseus for microbial control of the silverleaf whitefly, Bemisia argentifolii. Biol Control 17, 203-217.

Xiong, G.H., Xing, L.S., Lin, Z., Saha, T.T., Wang, C., Jiang, H., and Zou, Z. (2015). High throughput profiling of the cotton bollworm Helicoverpa armigera immunotranscriptome during the fungal and bacterial infections. BMC Genomics 16, 321.

Xu, L., Lou, Q., Cheng, C., Lu, M., and Sun, J. (2015). Gut-associated bacteria of Dendroctonus valens and their involvement in verbenone production. Microb Ecol 70, 1012-1023.

Yassine, H., Kamareddine, L., and Osta, M.A. (2012). The mosquito melanization response is implicated in defense against the entomopathogenic fungus Beauveria bassiana. PLoS Pathog 8, e1003029.

Zhao, L., Mota, M., Vieira, P., Butcher, R.A., and Sun, J. (2014). Interspecific communication between pinewood nematode, its insect vector, and associated microbes. Trends Parasitol 30, 299-308.

Zhao, L., Zhang, S., Wei, W., Hao, H., Zhang, B., Butcher, R.A., and Sun, J. (2013). Chemical signals synchronize the life cycles of a plant-parasitic nematode and its vector beetle. Curr Biol 23, 2038-2043.

Zou, Z., Evans, J.D., Lu, Z., Zhao, P., Williams, M., Sumathipala, N., Hetru, C., Hultmark, D., and Jiang, H. (2007). Comparative genomic analysis of the Tribolium immune system. Genome Biol 8, R177.

\section{SUPPORTING INFORMATION}

Table S1 FPKM values of DETs in intracellular signaling pathways of fungal infected M. alternatus

Table S2 Primers used in quantitative real-time PCR analysis

The supporting information is available online at http://life.scichina.com and https://link.springer.com. The supporting materials are published as submitted, without typesetting or editing. The responsibility for scientific accuracy and content remains entirely with the authors. 